\title{
Comparison of the impact between peak mean fluorescent intensity versus sum of mean fluorescent intensity value of donor specific anti-human leukocyte antigen antibody on the post- transplant clinical outcomes
}

\author{
Hyung Duk Kim ${ }^{1}$, Yohan Park ${ }^{2}$, Sang Hun Eum ${ }^{1}$, Hanbi Lee ${ }^{1}$, Eun Jeong Ko ${ }^{1}$, Chul Woo Yang ${ }^{1}$, Byung Ha Chung ${ }^{1}$ \\ 1Department of Internal Medicine-Nephrology, The Catholic University of Korea, Seoul St. Mary's Hospital, Seoul, Korea
${ }^{2}$ Department of Internal Medicine-Nephrology, Konyang University Hospital, Daejeon, Korea
}

Background: In this study, we investigated to verify which of the peak value of donor-specific human leukocyte antigen antibodies (DSA) mean fluorescent intensity (MFI) and sum of MFI had higher predictive value for antibody-mediated rejection (ABMR) in kidney transplant recipients (KTR).

Methods: Analysis was performed on 1,322 KTR in Seoul St. Mary's Hospital between 2009 and 2018. Of these, 511 patients did not require desensitization (control group) and 317 patients without DSA underwent desensitization for reasons such as positive crossmatch or PRA (no DSA group). There were 42 patients with one DSA (DSA 1 group) and 35 patients with two or more DSA (DSA 2 group). The effect of the DSA MFI value on ABMR was analyzed by cox proportional hazards analysis.

Results: The incidence of ABMR was 4.5\% in control group, 9.15\% in no DSA group, 19.05\% in DSA 1 group, and $37.1 \%$ in DSA 2 group $(\mathrm{P}=0.001)$. $T$ cell-mediated rejection, $\mathrm{BKV}$ nephropathy, $\mathrm{CNI}$ toxicity and graft failure did not differ significantly between groups. Sum of MFI's area under the receiver operating characteristic curve (AUC-ROC curve) for ABMR was 0.624, and peak MFI's AUC-ROC curve was $0.623(P=0.152)$. Compared with patients with both sum MFI and peak MFI were less than 1,000 , the hazard ratio for $A B M R$ of patients with sum MFI $>5,000$ was 2.79 , and the hazard ratio of patients with peak MFI $>5,000$ was 3.62 $(P=0.0016, P=0.0008$, respectively). However, when comparing the risk of ABMR between patients with sum MFI $>5,000$ and peak MFI $>5,000$, there was no significant difference. In DSA 2 group, ABMR occurred in three out of four patients with sum MFI $>5,000$ and peak $\mathrm{MFI}<5,000$, whereas eight out of 17 patients with peak MFI $>5,000$ developed ABMR.

Conclusions: In KTR with multiple DSA, both sum of MFI and peak MFI values over 5,000 showed increased risk of ABMR.

Corresponding author: Byung Ha Chung

E-mail: chungbh@catholic.ac.kr

(C) The Korean Society for Transplantation

This is an Open Access article distributed under the terms of the Creative Commons Attribution Non-Commercial License (http://creativecommons.org/licenses/by-nc/4.0/) which permits unrestricted non-commercial use, distribution, and reproduction in any medium, provided the original work is properly cited. 